\title{
VIABILIDADE TÉCNICA E ECONÔMICA DA REGENERAÇÃo DE COAGULANTES A PARTIR DE LODOS DE ESTAÇÕES DE TRATAMENTO DE ÁGUA
}

\section{TECHNICAL AND ECONOMICAL FEASIBILITY OF COAGULANT RECOVERY FROM WATER TREATMENT PLANT SLUDGES}

\section{JULIANA GARDENALLI DE FREITAS}

Engenheira Civil pela EPUSP. Mestre em Engenharia Hidráulica e Sanitária pela Escola Politécnica da Universidade de São Paulo. Engenheira da CETESB - Companhia de Tecnologia de Saneamento Ambiental

\section{SIDNEY SECKLER FERREIRA FILHO}

Professor Associado do Departamento de Engenharia Hidráulica e Sanitária da Escola Politécnica da Universidade de São Paulo em Regime de Dedicação Integral à Docência e a Pesquisa

\section{Roque Passos PIVELI}

Professor Doutor do Departamento de Engenharia Hidráulica e Sanitária da Escola Politécnica da Universidade de São Paulo em Regime de Dedicação Integral à Docência e a Pesquisa

Recebido: 16/12/04 Aceito: 09/03/05

\section{RESUMO}

Esse trabalho teve como objetivo avaliar a viabilidade técnica e econômica da regeneração de coagulantes pela acidificação de lodos de ETA's e a posterior aplicação dos coagulantes regenerados no tratamento de esgotos. Foram realizados ensaios em escala de bancada visando o estudo das condiçôes de regeneração, da qualidade do coagulante produzido e do lodo resultante. $\mathrm{O}$ coagulante regenerado teve desempenho satisfatório no tratamento de efluentes de reator UASB e foi observado que ocorreram reduções significativas de sólidos em suspensão nos lodos das ETA's. Com respeito à avaliação econômica, foi verificado que considerando as reduções de custos decorrentes da diminuição de lodo a ser tratado e disposto, o custo de produção do coagulante regenerado é muito próximo ao custo do coagulante comercial. Portanto, concluiu-se que hoje em dia essa tecnologia deve ser considerada como uma alternativa potencialmente viável dos pontos de vista técnico e econômico para o tratamento e reaproveitamento de lodo de ETA's.

PALAVRAS-CHAVE: Regeneração de coagulante, lodo de estação de tratamento de água, tratamento de esgotos.

\begin{abstract}
The primary goal of this work was to evaluate the technical and economical feasibility of coagulant recovery from acidification of water treatment plant sludges, and the use of recovered coagulants in wastewater treatment. Acidification bench tests were conducted in order to study the regeneration conditions, the recovered coagulant quality and the remaining sludge. The recovered coagulant had a satisfactory behavior in the treatment of UASB reactor effluent and was observed significantly suspended solids reductions in the water treatment plant sludges. On the economical evaluation it was verified that considering the costs decrease due to the sludge reduction, the recovered coagulant production cost was very similar to commercial coagulants cost. Thus, it was concluded that nowadays this technology must be considered as a potential alternative for the treatment and reuse for water treatment plant sludges, regarding to technical and economical aspects.
\end{abstract}

KEYWORDS: Coagulant recovery, water treatment plant sludge, wastewater treatment.

\section{INTRODUÇÃO}

Atualmente, um dos grandes problemas enfrentados pela Engenharia Sanitária e Ambiental é a destinação a ser dada aos resíduos gerados nas Estações de Tratamento de Água. No Brasil, esse assunto só vem recebendo atenção nos últimos anos (Ferreira Filho et al, 1997), apesar de ser um problema extremamen- te freqüente e que tende a se agravar devido ao maior consumo de água e geração de resíduos, por causa do crescimento populacional.

O tratamento de água visando torná-la potável gera uma quantidade de resíduos, que pode possuir características diferentes dependendo da concepção do sistema de tratamento. No Brasil, o sistema de tratamento mais utilizado é o cha- mado convencional, que gera dois tipos principais de resíduos. Um deles é a água de lavagem dos filtros, que, em boa parte das Estaçôes de Tratamento de Água (ETA's) vem recebendo tratamento e destinação adequados. Esse resíduo vem sendo reaproveitado, gerando uma economia aos sistemas de tratamento. A viabilidade econômica do reaproveitamento desse resíduo é o que tem tornado essa 
solução amplamente difundida (AWWA, 1991) e (Cornwell e Lee, 1994).

O outro resíduo gerado é o lodo resultante da decantação. Esse lodo é composto principalmente por hidróxidos de ferro e alumínio, que são gerados quando é feita a adição do coagulante no processo de tratamento de água (Petruzelli et al, 2000). Para esse resíduo ainda se está em busca de uma solução definitiva, sendo o seu tratamento e destinação final ainda grandes problemas ambientais. De acordo com a legislação brasileira, o lodo gerado nos decantadores é classificado como resíduo sólido, e, portanto, não pode ser disposto em corpos d'água superficiais (PROSAB, 2001).

Apesar disso, a maior parte das Estaçôes de Tratamento de Âgua (ETA's) do Brasil dispõe esse resíduo diretamente nos corpos d'água, uma prática que pode gerar uma série de danos ao meio ambiente, podendo promover impactos ambientais intensos (PROSAB, 2001). Essa situação se deve, em grande parte, à escassez de recursos e ao alto custo de implantação e operação dos sistemas de tratamento e disposição final de resíduos sólidos.

Atualmente, antes de se pensar na disposição e no tratamento de que os resíduos gerados em ETA's necessitam, devese pensar na utilização dos mesmos (AWWA, 1999). Assim, é de fundamental importância o desenvolvimento e aprimoramento de técnicas que viabilizem a utilização desses resíduos, bem como o seu tratamento e disposição adequada.

Uma das alternativas possíveis que poderia trazer benefícios para a sociedade em vários aspectos é a regeneração de coagulante, ou seja, a extração do coagulante utilizado no processo de tratamento de água, do lodo dos decantadores. Essa recuperação é realizada por meio de ajustes no $\mathrm{pH}$ do lodo, de forma a solubilizar os hidróxidos de ferro e alumínio.

A utilização dessa técnica pode trazer dois grandes benefícios: a recuperação de um recurso, o coagulante, e a minimização dos resíduos sólidos a serem tratados e dispostos no meio ambiente (ASCE, AWWA, EPA, 1996). Além dos benefícios ambientais, essa técnica pode trazer vantagens econômicas, propiciadas pela produção de um coagulante com custo reduzido e pela redução do custo de tratamento e disposição da fase sólida (AWWA, 1987).

Considerando-se que estudos anteriores levantaram problemas em relação à qualidade do coagulante regenerado, que pode conter concentração mais elevada de poluentes como metais pesados, a sua utilização em sistemas de abastecimento de água pode comprometer a qualidade d'água a ser distribuída à população. Portanto, é mais adequado que o coagulante regenerado seja utilizado em outros processos e não no tratamento de águas para abastecimento. Uma das alternativas para o uso do coagulante obtido pela regeneração é a aplicação em sistemas de esgotos sanitários (Masides et al, 1988).

\section{OBJETIVOS}

O objetivo desse trabalho foi estudar a viabilidade técnica e econômica da aplicação da tecnologia de regeneração de coagulante a partir de lodos de ETA's. Para isso, distinguem-se quatro objetivos principais a serem atingidos:

- Sugerir uma metodologia para verificação da viabilidade técnica da regeneração de coagulantes a partir de lodos gerados em estações de tratamento de água convencionais;

- Avaliar a qualidade do coagulante regenerado e discutir a sua aplicação em sistemas de tratamento de esgotos sanitários;

- Avaliar o impacto da regeneração do coagulante nas características do lodo resultante, em relação à redução de massa;

- Estimar do ponto de vista econômico a viabilidade da regeneração de coagulante, visando o seu emprego em sistemas de tratamento de esgotos.

\section{MATERIAIS E MÉTODOS}

Nos ensaios foram utilizados lodos dos decantadores da Estação de Tratamento de Água do Guaraú (ETA Guaraú) e da Estação de Tratamento de Rio Grande (ETA Rio Grande), ambas localizadas na Região Metropolitana de São Paulo e operadas pela SABESP. Essas ETA's foram escolhidas por possuírem sistemas de remoção mecanizados para remoção do lodo, o que facilita a sua coleta. As principais características dessas ETA's estão indicadas na Tabela 1 .

\section{Regeneração do coagulante}

A regeneração foi realizada pela acidificação e filtração dos lodos, conforme procedimento básico apresentado na seqüência:

- um béquer de $500 \mathrm{~mL}$ é preenchido com o lodo a ser ensaiado;
- é adicionado o ácido sulfúrico ao lodo e mantido sobre agitação por um período pré-determinado. Durante essa etapa é monitorado o $\mathrm{pH}$ da mistura ao longo do tempo;

- depois de completado o tempo de ensaio, são retiradas duas amostras;

- uma amostra é filtrada em papel de filtro $n^{\circ} 40$ ou no 42 e posteriormente em membrana $0,45 \mu \mathrm{m}$ e submetida à análise de ferro ou alumínio e em alguns casos outros metais;

- a outra amostra é utilizada para a determinação das concentrações de sólidos em suspensão totais (SST), fixos (SSF) e voláteis (SSV).

O ácido utilizado foi o ácido sulfúrico $5 \mathrm{~N}$, por ser o mais utilizado nas investigaçôes apresentadas na literatura, e por apresentar custo inferior ao ácido clorídrico, que também foi considerado eficiente em outras pesquisas (AWWA, 1991).

Buscando estudar as condiçôes de regeneração foram realizados ensaios variando os principais parâmetros que interferem na regeneração, o tempo e o $\mathrm{pH}$ (ASCE, 1996); (AWWA, 1991) e (Masschelein et al, 1985). As condições adotadas em cada ensaio estão indicadas nas Tabelas 2 e 3 . O objetivo da realização de ensaios com diferentes tempos de acidificação (ensaios R4, R5, G4 e G5) foi estudar a cinética da reação e determinar um tempo que proporcionasse resultados adequados, levando em consideração também que, quanto maior o tempo, maior é o custo com o reator para a acidificação.

Os ensaios R6 e G6 tiveram como objetivo avaliar qual o $\mathrm{pH}$ ótimo para os lodos que estão sendo estudados. Além disso, o estudo do efeito do $\mathrm{pH}$ também é importante do ponto de vista econômico, pois esse valor irá determinar a quantidade de ácido necessária para a operação do sistema.

\section{Caracterização do coagulante obtido}

Em todos os ensaios o coagulante regenerado foi analisado para ferro e alumínio no IPEN, no Centro de Química e Meio Ambiente (CQMA), em espectrômetro de emissão com fonte de plasma induzido. Em sete amostras de coagulantes regenerados também foram analisadas as concentrações de outros metais além de alumínio e ferro, pois os metais são um dos principais grupos de contaminantes que podem ser encontra- 
dos em coagulantes regenerados. Os metais analisados foram manganês, cromo, níquel, chumbo, zinco, cádmio e cobre.

Também foram realizados ensaios de bancada tipo jar-test simulando processos físico-químicos para remoção de fósforo de efluentes de reatores UASB. Os ensaios foram realizados com o coagulante regenerado e com coagulante comercial, permitindo uma análise comparativa. O coagulante comercial utilizado foi sulfato férrico. Somente foi utilizado o coagulante regenerado a partir do lodo da ETA Rio Grande pois a ETA Guaraú vem utilizando no tratamento coagulantes a base de ferro e alumínio simultaneamente, e, portanto, o coagu- lante regenerado também apresentava concentraçôes significativas dos dois compostos, impossibilitando uma análise comparativa entre o coagulante regenerado e o coagulante comercial.

Para a realização desses ensaios foram realizados 04 ensaios de acidificação (R7, R8, R9 e R10) com o objetivo de gerar um volume de coagulante regenerado suficiente. Esses ensaios foram realizados com tempo de mistura de $10 \mathrm{mi}$ nutos e $\mathrm{pH}$ próximos de 2 . Os ensaios tipo jar-test foram realizados com uma etapa de mistura rápida de $30 \mathrm{~s}$, com rotação de 300rpm, floculação em três etapas de 5 min com gradientes de velocidade decrescentes (60, 40 e 20rpm) e sedi- mentação de 6 a 18 min. As dosagens de coagulante utilizadas estão apresentadas na Tabela 4.

Os parâmetros analisados nas amostras coletadas em todos os jarros e no efluente do UASB foram: turbidez, SST, SSF e SSV, DQO, pH e alcalinidade. Além disso, em cada ensaio, duas amostras que apresentaram melhores resultados para cada coagulante (resultados de DQO) e no efluente do UASB também foram realizadas determinaçôes de fósforo (total e solúvel) e DBO. Todas as análises foram realizadas seguindo os procedimentos determinados em Standard Methods for the Examination of Water and Wastewater (APHA, AWWA e WEF, 1995).
Tabela I - Características das ETA's

\begin{tabular}{ccc}
\hline Parâmetro & ETA Guaraú & ETA Rio Grande \\
\hline \multicolumn{2}{c}{ Características da água bruta } \\
turbidez $(\mathrm{UNT})$ & $3,1^{1}$ & $4,5^{2}$ \\
cobre $(\mathrm{mg} / \mathrm{L})^{1}$ & $\mathrm{Nd}$ & 0,03 \\
manganês $(\mathrm{mg} / \mathrm{L})^{1}$ & 0,04 & 0,06 \\
Características do processo & 5,3 \\
vazão $\left(\mathrm{m}^{3} / \mathrm{s}^{3}\right.$ & 33 & sulfato férrico \\
coagulante ${ }^{2}$ & sulfato de \\
& alumínio \\
sulfato férrico
\end{tabular}

nd: não detectado

Fontes consultadas: ${ }^{1} \mathrm{CETESB}, 2004$ (valores médios de 6 análises nos pontos de captação dos reservatórios), ${ }^{2}$ SABESP ETA Rio Grande e ETA Guaraú, ${ }^{3}$ SABESP, 2004 b

Tabela 3 - Condições de realização dos ensaios de regeneração com lodo da ETA Guaraú

\begin{tabular}{ccc}
\hline Ensaio & $\begin{array}{c}\text { pH de } \\
\text { acidificação }\end{array}$ & $\begin{array}{c}\text { Tempo de } \\
\text { acidificação }(\mathrm{min})\end{array}$ \\
\hline G1 & 2,4 & 30 \\
G2 & 2,3 & 30 \\
G3 & 2,4 & 30 \\
G4A & 2,5 & 15 \\
G4B & 2,5 & 30 \\
G4C & 2,4 & 45 \\
G5A & 2,3 & 05 \\
G5B & 2,4 & 10 \\
G5C & 2,4 & 15 \\
G6A & 2 & 10 \\
G6B & 3 & 10 \\
G6C & 3,6 & 10 \\
\hline
\end{tabular}

Tabela 2 - Condições de realização dos ensaios de regeneração com lodo da ETA Rio Grande

\begin{tabular}{ccc}
\hline Ensaio & $\begin{array}{c}\text { pH de } \\
\text { acidificação }\end{array}$ & $\begin{array}{c}\text { Tempo de } \\
\text { acidificação (min) }\end{array}$ \\
\hline R1 & 2,4 & 30 \\
R2 & 2,3 & 30 \\
R3 & 2,2 & 30 \\
R4A & 2,4 & 15 \\
R4B & 2,4 & 30 \\
R4C & 2,4 & 45 \\
R5A & 2,5 & 05 \\
R5B & 2,6 & 10 \\
R5C & 2,6 & 15 \\
R6A & 2 & 10 \\
R6B & 2,6 & 10 \\
R6C & 5 & 10 \\
\hline
\end{tabular}

Tabela 4 - Condições de realização dos ensaios tipo jar-test

\begin{tabular}{|c|c|}
\hline Ensaio & $\begin{array}{l}\text { Dosagens } \\
(\mathrm{mgFe} / \mathrm{L})\end{array}$ \\
\hline R7 & $\begin{array}{c}10,30,60 \text { (com.) } \\
5,14,28 \text { (reg.) }\end{array}$ \\
\hline $\mathrm{R} 8$ & $10,25,40$ (com. e reg.) \\
\hline R9 & $\begin{array}{l}10,30,50 \text { (com.) } \\
20,61,101 \text { (reg.) }\end{array}$ \\
\hline $\mathrm{R} 10$ & $\begin{array}{c}10,40,70 \text { (com.) } \\
5,19,33 \text { (reg.) }\end{array}$ \\
\hline
\end{tabular}




\section{Caracterização do lodo resultante}

Para a avaliação do lodo resultante após a acidificação, foi considerada a redução da massa total. A redução total de massa foi avaliada pela variação da concentração de sólidos em suspensão no lodo acidificado $\left(\mathrm{SS}_{\mathrm{A}}\right)$ e não acidificado $\left(\mathrm{SS}_{0}\right)$, conforme a equação (1). Esse parâmetro foi avaliado em todos os ensaios realizados.

redução $=\frac{S S_{0}-S S_{A}}{S S_{0}}$

\section{APRESENTACÃO E DISCUSSÃO DOS RESULTADOS EXPERIMENTAIS}

\section{Regeneração do coagulante}

Os resultados dos ensaios R4 e G4, realizados com tempo de mistura variável em relação às concentrações de ferro e alumínio estão apresentados na Figura 1.

Pode-se observar que as concentraçôes de $\mathrm{Al}$ e $\mathrm{Fe}$ nos dois lodos cresceram significativamente nos primeiros $15 \mathrm{mi}$ nutos e permaneceram praticamente constantes nos minutos seguintes.

A redução da concentração de sólidos em suspensão apresentou comportamento semelhante, sendo que as reduções mais significativas da concentração, 30 e $55 \%$ para os lodos da ETA Guaraú e Rio Grande, respectivamente ocorreram após quinze minutos.

Para avaliar como ocorria a variação da concentração de sólidos e da concentração de $\mathrm{Al}$ e Fe nos 15 minutos iniciais, foram realizados os ensaios $\mathrm{R} 5$ e G5.

Nesses ensaios, a redução da concentração de sólidos em suspensão ocorreu principalmente nos 5 minutos iniciais, indicando que a reação é rápida. Nesse período foi verificada redução de $78,6 \%$ e $73,37 \%$ da máxima observada durante todo o período de ensaio para os lodos da ETA Rio Grande e ETA Guaraú, respectivamente.

Esse mesmo comportamento foi observado no ensaio $\mathrm{R} 5$ em relação à concentração de ferro. Nesse ensaio, a concentração se elevou de zero a 278,6 mg/L nos cinco minutos iniciais e após esse período a concentração de ferro permaneceu praticamente constante. Já no ensaio G5 a concentração de ferro apresentou maior aumento entre 5 e 10 minutos.

Portanto, foi concluído que a reação é rápida, sendo que nos ensaios R5 e G5

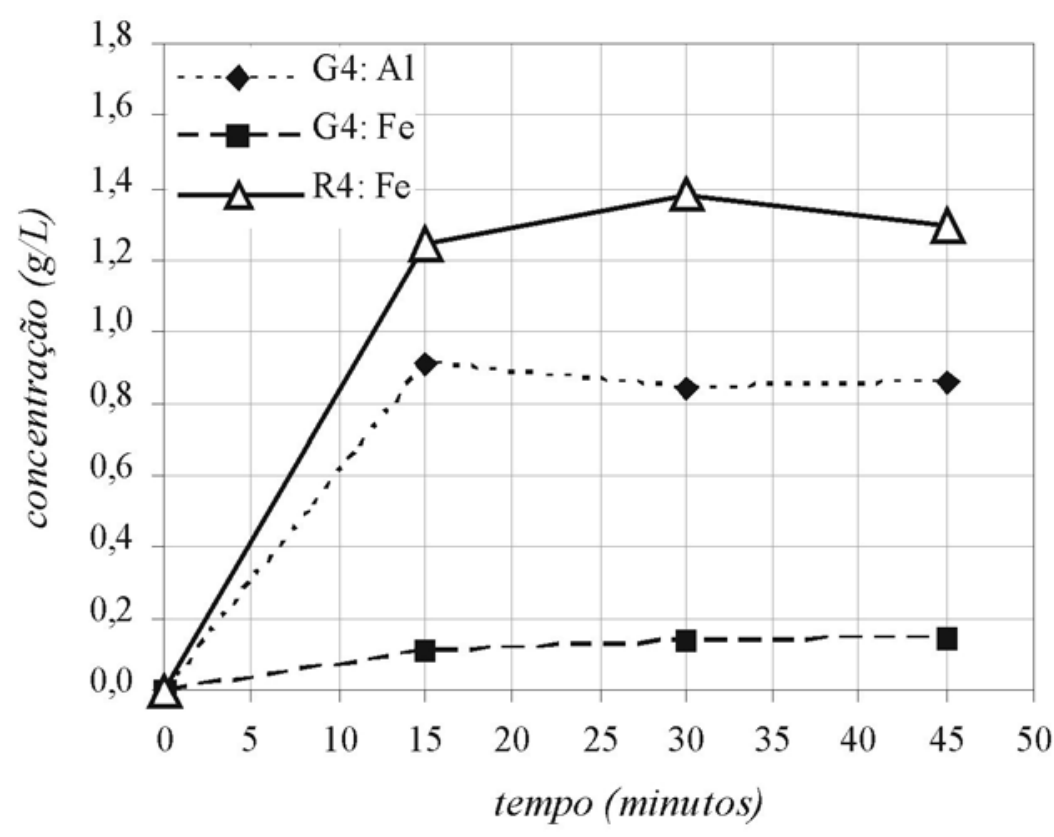

Figura I - Ensaios R4 e G4: Efeito do tempo de mistura nas concentrações de alumínio e ferro

verificou-se que a maior parte da reação ocorreu nos cinco minutos iniciais. Optou-se por fixar o tempo ótimo em 10 minutos considerando que esse período de tempo foi suficiente para que ocorresse a reação em todos os ensaios.

Além disso, esse tempo foi considerado adequado do ponto de vista de instalaçōes, não implicando em volumes muito elevados para o reator. Apesar do tempo de 5 minutos ter sido adequado no ensaio de bancada R5, não foi adotado tempo inferior a 10 minutos, pois isso não ocorreu em todos os ensaios (como no ensaio G5) e em instalações reais provavelmente não se terá condições de mistura iguais às que são obtidas em escala de bancada.

Os resultados obtidos nos ensaios com diferentes valores de $\mathrm{pH}$ (R6 e G6) estão apresentados na Figura 2. Observando essa Figura pode-se perceber que os melhores resultados (maior concentração dos metais de interesse) são obtidos com $\mathrm{pH}$ próximo de 2 , ocorrendo uma queda significativa da eficiência, de 71 a $90 \%$ para as concentraçôes de $\mathrm{Fe}$ e $\mathrm{Al}$ com um pequeno aumento do $\mathrm{pH}$, de 2 a valores entre 2,6 e 3. Esse mesmo efeito foi observado na redução da concentração de sólidos em suspensão, tendo sido observada queda de eficiência de 67 a $97 \%$ para o mesmo aumento de $\mathrm{pH}$.

Além disso, durante a execução dos ensaios foi observado que o volume de ácido necessário para se atingir valores de
$\mathrm{pH}$ inferiores a 2 era muito elevado, o que inviabilizaria a acidificação com valores de $\mathrm{pH}$ menores que 2 .

Portanto, foi concluído que o $\mathrm{pH}$ ideal para a acidificação é em torno de 2 , uma vez que com esse valor foram obtidos bons resultados. Além disso, foi verificada a inadequação da acidificação com $\mathrm{pH}$ superior a 2 , devido à queda significativa da eficiência, e inferior a 2 devido à grande quantidade de ácido necessária para atingir esse valor.

Da mesma forma que foi observado em relação ao tempo de acidificação, o comportamento dos lodos da ETA Rio Grande e da ETA Guaraú em relação ao $\mathrm{pH}$ foi semelhante, sendo que para os lodos oriundos das duas ETA's estudadas o $\mathrm{pH}$ ideal para acidificação foi o mesmo.

Uma relação importante do ponto de vista operacional e econômico é a relação entre a massa de ácido adicionada e o pH do lodo. Na Figura 3 são apresentados os resultados obtidos em relação a esses parâmetros.

As reaçōes esperadas no processo de regeneração ácida são as apresentadas nas equaçōes 2 e 3 (Bishop et al, 1987) e (Gonçalves et al, 1997).

Pela Figura 3, pode-se observar que, em geral, a acidificação do lodo da ETA Guaraú demandou menos ácido por massa de sólidos que o lodo da ETA Rio Grande. Considerando a estequiometria das reaçôes, seria esperado que no lodo 


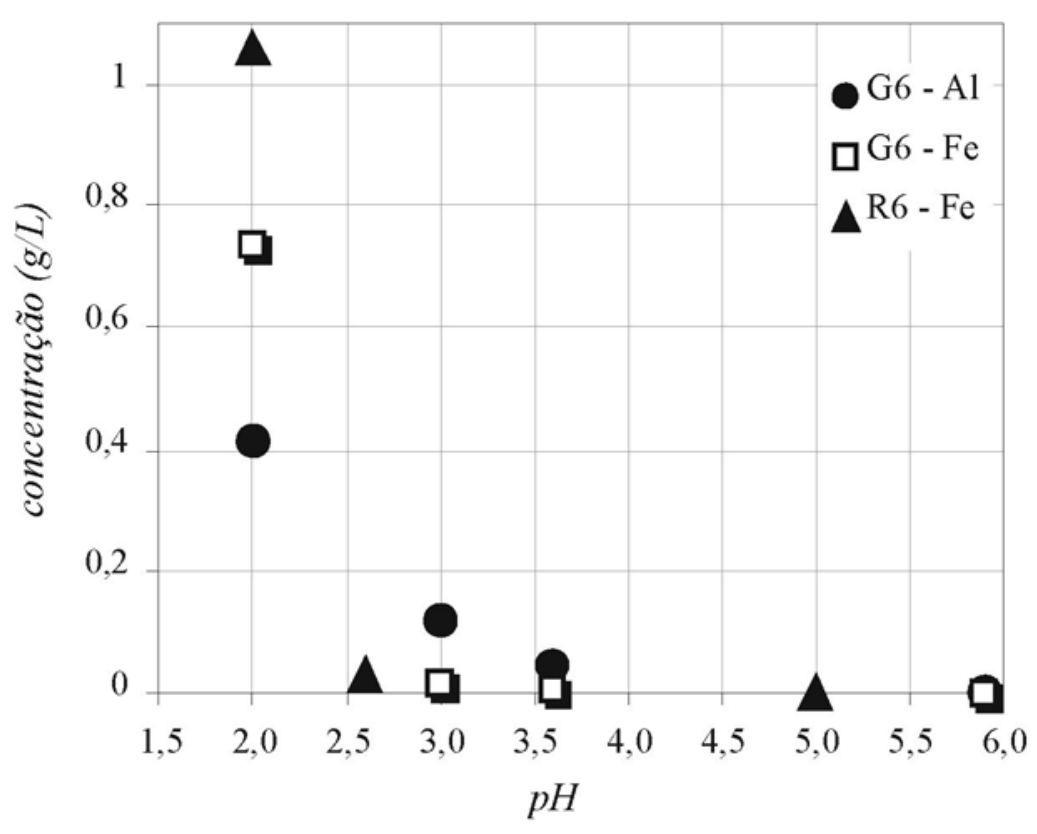

Figura 2 - Ensaios G6 e R6: Efeito do pH nas Concentrações de Fe e Al

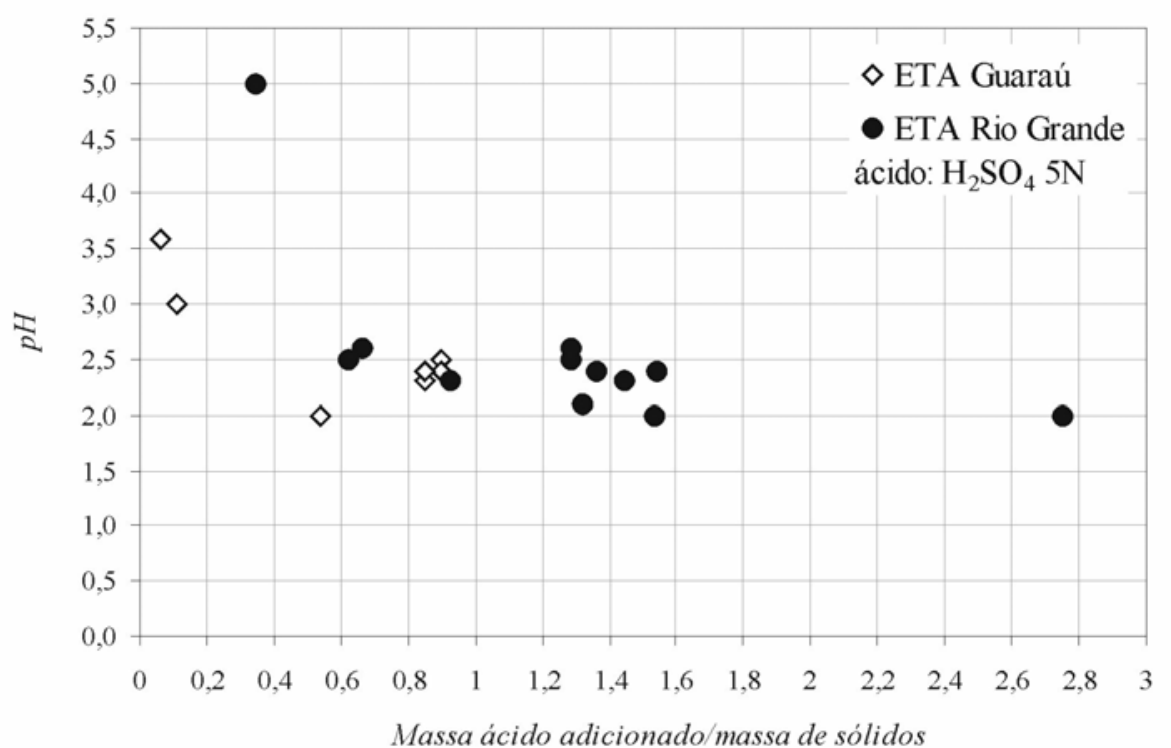

Figura 3 - Ensaios G6 e R6: Efeito do pH nas Concentrações de Fe e Al

da ETA Guaraú a massa de ácido necessária fosse maior, uma vez que nesse lodo deve haver a presença de hidróxidos de alumínio em conjunto com os de ferro, e pela relação estequiométrica, esses hidróxidos consomem uma massa maior de ácido.

$2 \mathrm{Al}(\mathrm{OH})_{3(\mathrm{~S})} \cdot 3 \mathrm{H}_{2} \mathrm{O}+3 \mathrm{H}_{2} \mathrm{SO}_{4}+2 \mathrm{H}_{2} \mathrm{O} \rightarrow \mathrm{Al}_{2}\left(\mathrm{SO}_{4}\right)_{3} \cdot 14 \mathrm{H}_{2} \mathrm{O}$

$2 \mathrm{Fe}(\mathrm{OH})_{Y \mathrm{~S})} \cdot 3 \mathrm{H}_{2} \mathrm{O}+3 \mathrm{H}_{2} \mathrm{SO}_{4}+2 \mathrm{H}_{2} \mathrm{O} \rightarrow \mathrm{Fe}_{2}\left(\mathrm{SO}_{4}\right)_{3} \cdot 14 \mathrm{H}_{2} \mathrm{O}$
Portanto, o que deve estar influindo na relação entre a quantidade de ácido e o pH são outros compostos presentes no lodo que também exercem demanda pelo ácido além dos hidróxidos.

É interessante observar que pelas reações apresentadas, a quantidade de ácido que necessitaria ser adicionada é de 1,5 mol por mol de alumínio ou ferro. Essa relação pode ser colocada em termos de massa, resultando em $1,1 \mathrm{~kg} \mathrm{H}_{2} \mathrm{SO}_{4} / \mathrm{kg} \mathrm{Al}(\mathrm{OH})_{3} .3 \mathrm{H}_{2} \mathrm{O}$, no caso de sais de alumínio, ou
$0,91 \mathrm{~kg} \mathrm{H}_{2} \mathrm{SO}_{4} / \mathrm{kg} \mathrm{Fe}(\mathrm{OH})_{3} \cdot 3 \mathrm{H}_{2} \mathrm{O}$, no caso de sais de ferro.

Comparando os valores obtidos para o lodo da ETA Rio Grande da relação entre a massa de ácido adicionado e a massa de sólidos com os valores previstos pela estequiometria da reação, observa-se que em geral os valores para a obtenção de $\mathrm{pH}$ relativamente baixos (que propiciaram maiores concentraçóes de ferro, e, portanto, mais próximos da situação de solubilização total dos hidróxidos metálicos, o que éassumido nas equaçōes apresentadas), situaram-se acima do valor previsto de $0,91 \mathrm{~kg} \mathrm{H}_{2} \mathrm{SO}_{4} / \mathrm{kg} \mathrm{Fe}(\mathrm{OH})_{3}$. $3 \mathrm{H}_{2} \mathrm{O}$. Isso também é um indicador da presença significativa de outros compostos no lodo que exercem demanda pelo ácido.

Ressalta-se que nos ensaios a relação foi estabelecida entre a massa de ácido e a massa de sólidos em suspensão totais, não havendo como distinguir quanto dessa massa é realmente de hidróxidos e quanto é de partículas provenientes da água bruta. Portanto, as relações entre as massas de ácido adicionado e as massas de hidróxidos são ainda maiores que as indicadas na Figura 3.

$\mathrm{Na}$ maior parte dos ensaios observou-se que qualitativamente os resultados de redução da concentração de sólidos e concentração de metais ( $\mathrm{Fe}$ ou $\mathrm{Al}$ ) variavam de forma semelhante com as condiçôes de ensaio, indicando que esses parâmetros estão diretamente relacionados. Considerando isso, buscou-se uma relação entre os valores da concentração de sólidos em suspensão e concentração de Al ou $\mathrm{Fe}$, estabelecendo-se relaçóes lineares entre SST e a concentração de $\mathrm{Al}$ ou Fe.

A Figura 4 apresenta os resultados obtidos para o lodo da ETA Rio Grande, onde pode-se observar que os dados apresentaram distribuição aproximadamente linear. Esse comportamento, caracterizado por uma relação linear entre a redução da concentração de sólidos em suspensão e a concentração no coagulante pode ser empregado no caso da eventual utilização em escala real da regeneração de coagulante.

\section{Qualidade do coagulante}

A avaliação do coagulante regenerado foi feita de forma comparativa, avaliando o coagulante regenerado em relação aos coagulantes comerciais. Os aspectos considerados para a avaliação comparativa foram três: as concentrações de metais; as concentrações de contaminantes e as eficiências dos dois tipos de 
coagulantes em ensaios pilotos para tratamento físico-químico de efluente de reator UASB.

Em relação à concentração de ferro, nos ensaios realizados com lodo da ETA Rio Grande e condições próximas às determinadas como ideais, ou seja, $\mathrm{pH}$ próximo de 2 e tempo de mistura de $10 \mathrm{mi}$ nutos, foram determinados os valores máximo, mínimo, média e o desvio padrão das concentrações de Fe obtidas. Os valores estão colocados na Tabela 5, juntamente com valores de coagulantes comerciais.

Por esses resultados pode-se perceber claramente que as concentraçôes obtidas no coagulante regenerado são muito inferiores, da ordem de 100 vezes, às exigidas por consumidores e atingidos por fabricantes de sulfato férrico destinado ao uso em tratamento de águas para abastecimento, uso que não está sendo considerado para o coagulante regenerado.

Em relação aos contaminantes, o composto observado em maiores concentraçôes foi o magnésio, com valores variando de 1,93 a 22,1 mg/L. Para alguns metais, como o cobre, foi observada uma relação entre a fonte do lodo utilizado e as concentraçôes encontradas. Isso pode ser observado na Figura 5.

Uma justificativa possível para isso é que essas diferenças sejam causadas pelas diferenças de qualidade dos mananciais. Outra justificativa possível seria que a contaminação encontrada no coagulante regenerado não é oriunda da água bruta, e sim dos coagulantes utilizados no tratamento. Com isso, as diferenças encontradas poderiam ter sido geradas pelas diferenças na qualidade dos coagulantes utilizados nas duas ETA's.

O mais provável é que as duas justificativas apresentadas ocorram simultaneamente, influindo nas concentrações encontradas nos coagulantes regenerados.

Para comparar os resultados obtidos com os valores dos coagulantes comerciais, foi feita uma relação entre a concentração dos contaminantes e a concentração de Fe. Foi observado que as concentraçôes de contaminantes em geral são da mesma ordem de grandeza no coagulante regenerado e no estabelecido para os coagulantes comerciais. O único contaminante que sempre apareceu em maiores concentraçóes no coagulante regenerado foi o alumínio. Portanto, provavelmente não existiriam restrições ao uso do coagulante regenerado para tratamento de esgotos sanitários, pois as concentraçôes estão muito próximas aos valores es-

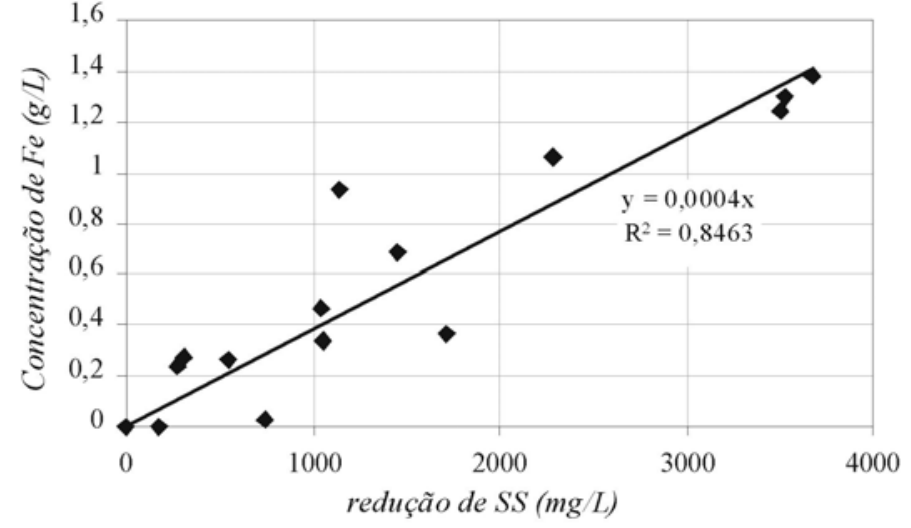

Figura 4 - Ensaios com Lodo da ETA Rio Grande: Redução de SS X Concentração de Fe

Tabela 5 - Comparação entre as concentrações de Fe no coagulante regenerado e no coagulante comercial

\begin{tabular}{ccc}
\hline & $\begin{array}{c}\text { Coagulante } \\
\text { regenerado }\end{array}$ & $\begin{array}{c}\text { Coagulante } \\
\text { comercial }\end{array}$ \\
\hline Mínima & $0,0238 \%$ & $12,24 \%$ \\
Máxima & $0,1382 \%$ & $10,84 \%$ \\
Média & $0,0710 \%$ & $11,73 \%$ \\
Desvio padrão & $0,0426 \%$ & $0,394 \%$ \\
\hline
\end{tabular}

Obs.: foi considerada densidade de $1000 \mathrm{~g} / \mathrm{L}$

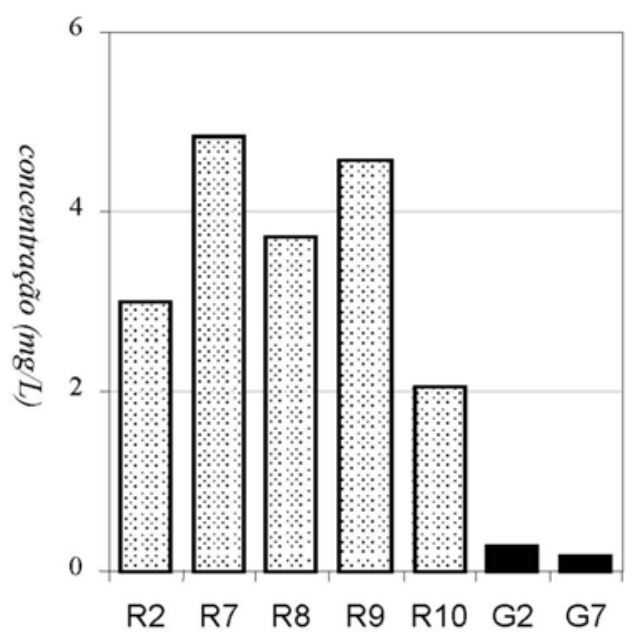

\section{Figura 5 - Concentrações de cobre nos coagulantes regenerados}

tabelecidos para o uso em tratamento de águas para abastecimento.

Nos ensaios tipo jar-test realizados com efluente de reator UASB o desempenho do coagulante regenerado foi muito similar ao do coagulante comercial em relação aos parâmetros monitorados, como exemplificado com os resultados do ensaio $\mathrm{R} 8$ para os parâmetros $\mathrm{DQO}$ e fós- foro total, nas Figuras 6 e 7, respectivamente. Com os dois coagulantes foram obtidos resultados satisfatórios, com reduçōes significativas de SST, DQO, $\mathrm{DBO}$, turbidez, fósforo total e solúvel, da ordem de $90 \%$ em boa parte dos ensaios.

Além disso, foi possível verificar visualmente a similaridade entre os jarros 


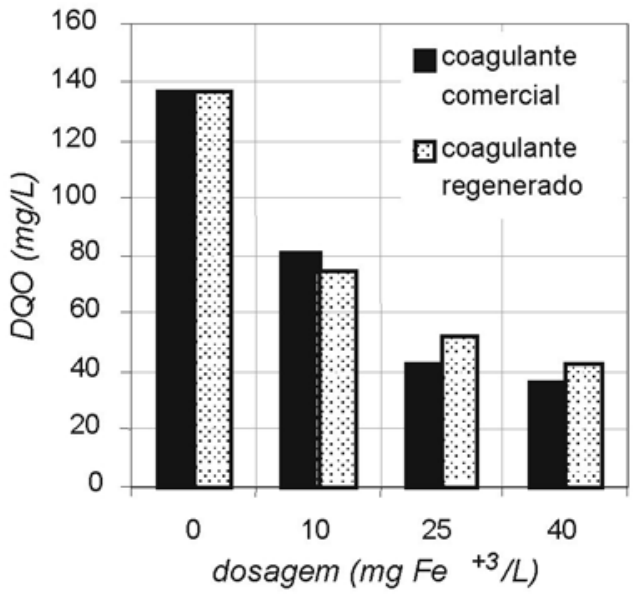

Figura 6 - Resultado do ensaio jar-test $R 8$ para DQO

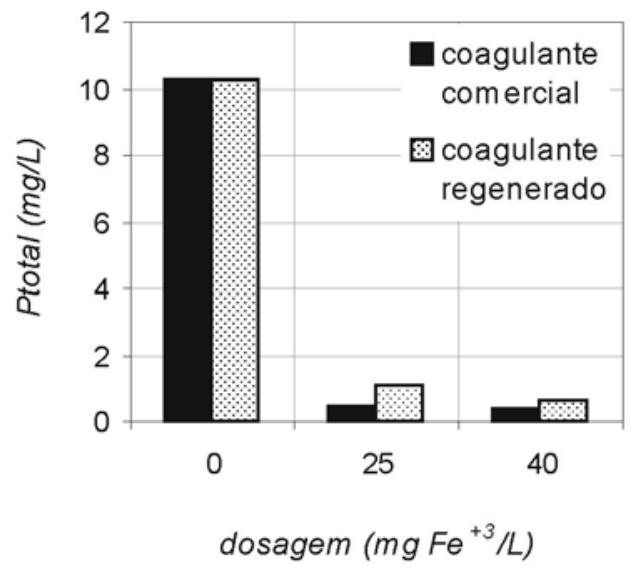

Figura 7 - Resultado do ensaio jar-test $R 8$ para fósforo total onde foram aplicados coagulantes regenerado e comercial com as mesmas concentrações. Isso foi verificado nas diferentes etapas do ensaio, na coagulação, floculação, e na sedimentação, quando foram observados a velocidade de sedimentação, a aparência do sobrenadante e o volume de lodo gerado.

\section{Caracterização do lodo resultante}

Considerando os ensaios realizados com condições similares às que foram definidas como ótimas ( $\mathrm{pH}$ próximo de $2 \mathrm{e}$ tempo de mistura mínimo de 10 minutos), foram calculados os valores médios de redução da concentração de sólidos em suspensão. Para o lodo da ETA Guaraú a redução média da concentração de sólidos em suspensão foi de $28 \%$, com desvio padrão de 5\%. Já para o lodo da ETA Rio Grande o valor médio foi $53 \%$, com desvio padrão de $14 \%$. Os resultados obtidos para o lodo da ETA Rio Grande foram comparados com os valores teóricos esperados, obtidos pela aplicação da Equação (4).

$$
\text { redução }(\%)=\frac{2,88 \cdot \mathrm{D}_{\mathrm{Fe}}}{2,88 \cdot \mathrm{D}_{\mathrm{Fe}}+\mathrm{SST}+\mathrm{A}} \cdot 100
$$

onde:

$\mathrm{D}_{\mathrm{Fe}}$ : dosagem de sais de ferro, expressa como Fe em mg/L;

SST: concentração de sólidos em suspensão totais na água em mg/L; zados

A: sólidos de outros produtos utili-

Os resultados obtidos nos ensaios R4 e R5 estão apresentados na Tabela 6. Para o ensaio R4 os valores de redução dos SST foram pouco inferiores aos valo- res teóricos calculados. Isso era esperado, uma vez que a equação utilizada se baseia no conceito que todo o ferro adicionado pelo coagulante se precipita na ETA e com a acidificação todo o ferro é solubilizado. Dificilmente isso acontece na prática. Além disso, no cálculo o valor do SST na água bruta foi estimado, podendo haver diferenças.

Já no ensaio $\mathrm{R} 5$ os valores de redução de massa observados foram muito inferiores ao esperado, um indício que a acidificação não ocorreu de forma adequada. Isso pode ter acontecido por alguns fatores, como $\mathrm{pH}$ relativamente elevado e tempos de mistura menores.

\begin{tabular}{ccc}
\multicolumn{3}{c}{$\begin{array}{c}\text { Tabela } 6 \text { - Redução de SS } \\
\text { observada e esperada }\end{array}$} \\
\hline Ensaio & $\begin{array}{c}\text { Redução } \\
\text { observada }\end{array}$ & $\begin{array}{c}\text { Redução } \\
\text { calculada }\end{array}$ \\
\hline R4-B & $55,08 \%$ & \\
R4-C & $57,74 \%$ & $65,9 \%$ \\
R4-D & $55,38 \%$ & \\
R5-B & $10,18 \%$ & \\
R5-C & $8,95 \%$ & $62,6 \%$ \\
R5-D & $17,90 \%$ & \\
\hline
\end{tabular}

\section{AVALIACÃO ECONÔMICA}

A avaliação econômica se baseou na consideração de implantação do sistema de regeneração em uma ETA já existente. Esse sistema é composto basicamente por um reator para acidificação do lodo. A diferença do custo de operação da ETA, decorrente da unidade de acidificação, foi utilizado para calcular o custo do coagulante regenerado. Esse custo foi comparado com o custo de coagulantes comerciais disponíveis no mercado e, dessa maneira, foi avaliado se existem benefícios econômicos na realização da regeneração de coagulante.

Foi considerado que as condiçôes de operação da ETA são semelhantes as existentes na ETA Rio Grande (ETA convencional, utilizando sulfato férrico como coagulante) e que as condiçóes de regeneração são as obtidas nos ensaios realizados em laboratório. $\mathrm{O}$ uso previsto para o coagulante regenerado foi o tratamento de esgotos.

Foram considerados dois cenários, detalhados nos itens abaixo:

- Cenário 1: Foi assumido como hipótese que uma outra empresa receba gratuitamente o lodo da ETA para fazer a regeneração e retorne para ETA os resíduos restantes, não sendo responsável pelo tratamento e disposição desses resíduos. Esse cenário calcula a viabilidade para essa empresa realizar esse serviço, e, portanto, nesse cenário não são consideradas as reduções de custos decorrentes da redução de lodo.

- Cenário 2: Foram consideradas as reduçóes de custo de transporte e disposição final resultantes da massa e volume de lodo. Esse cenário representa, por exemplo, que a própria operadora da ETA implante o sistema de regeneração de coagulantes.

Os custos dos coagulantes regenerados foram calculados pela equações 5 e 6.

Cenário 1:

Custo $=\frac{\mathrm{Q}_{\mathrm{a}} \cdot \mathrm{A}}{\mathrm{Q}_{\mathrm{C}} \cdot \mathrm{C}}$ 
Cenário 2:

Custo $=\frac{\mathrm{Q}_{\mathrm{a}} \cdot \mathrm{A}-(\mathrm{T}+\mathrm{D}) \cdot\left(\mathrm{M}_{\mathrm{LI}}-\mathrm{M}_{\mathrm{LF}}\right)}{\mathrm{Q}_{\mathrm{C}} \cdot \mathrm{C}}$

onde:

Custo: custo de produção do coagulante regenerado $(\mathrm{R} \$ / \mathrm{kg} \mathrm{Fe})$;

$\mathrm{Q}_{\mathrm{a}}$ : vazão de ácido utilizado na regeneração (ton / dia);

A: custo do ácido ( $\mathrm{R}$ / ton);

T: custo de transporte do lodo (R\$/ton);

D: custo de disposição do lodo (R\$/ton);

$\mathrm{M}_{\mathrm{LI}}:$ massa de lodo gerada antes da instalação do sistema de regeneração (ton/ dia);

$\mathrm{M}_{\mathrm{LF}}:$ massa de lodo gerada após a instalação do sistema de regeneração (ton/dia);

$\mathrm{Q}_{\mathrm{C}}$ : vazão de coagulante regenerado produzida ( $\left.\mathrm{m}^{3} / \mathrm{dia}\right)$;

C: concentração de ferro no coagulante regenerado $\left(\mathrm{kg} \mathrm{Fe} / \mathrm{m}^{3}\right)$;

A variável $A$ foi obtida com um fabricante de ácido sulfúrico, de $\mathrm{R} \$ 372,70 /$ ton, e para a variável $T+D$ foi obtido valor de $\mathrm{R} \$ 60,00 /$ ton (SABESP, 2004a). As demais variáveis foram obtidas por balanço de massa a partir dos dados de operação da ETA Rio Grande e com dados obtidos na parte experimental. Todos os valores de custo foram obtidos para o mês de maio de 2004.

Os custos obtidos para o coagulante regenerado foram 2,41 e 1,63 R $\$ / \mathrm{kgFe}$ para os cenários 1 e 2 , respectivamente. O custo do coagulante comercial foi fornecido pela Kemwater Brasil, de 1,74 R $\$ / \mathrm{kgFe}$. Esse valor é $28 \%$ inferior ao calculado para o Cenário 1 e 6\% maior que o valor obtido para o coagulante regenerado no Cenário 2.

Portanto, no Cenário 1, que não considerou as vantagens econômicas decorrentes da redução de lodo, a regeneração nas condições assumidas não seria viável economicamente. Já no Cenário 2, que considera o reflexo econômico da redução do lodo, o custo do coagulante regenerado foi inferior ao do coagulante comercial, mas os valores foram muito próximos, indicando que a operação do sistema de regeneração de coagulante tem o potencial de ser viável economicamente.

Considerando queos dois cenários apresentaram resultados distintos, uma variável que claramenteinterfere na viabilidade econômica é o custo de transporte e disposição do lodo. Alterandoessevalor, obtém-seosvalores decusto do coagulante regenerado para os Cenários 1 e2, conforme indicado na Figura 8.

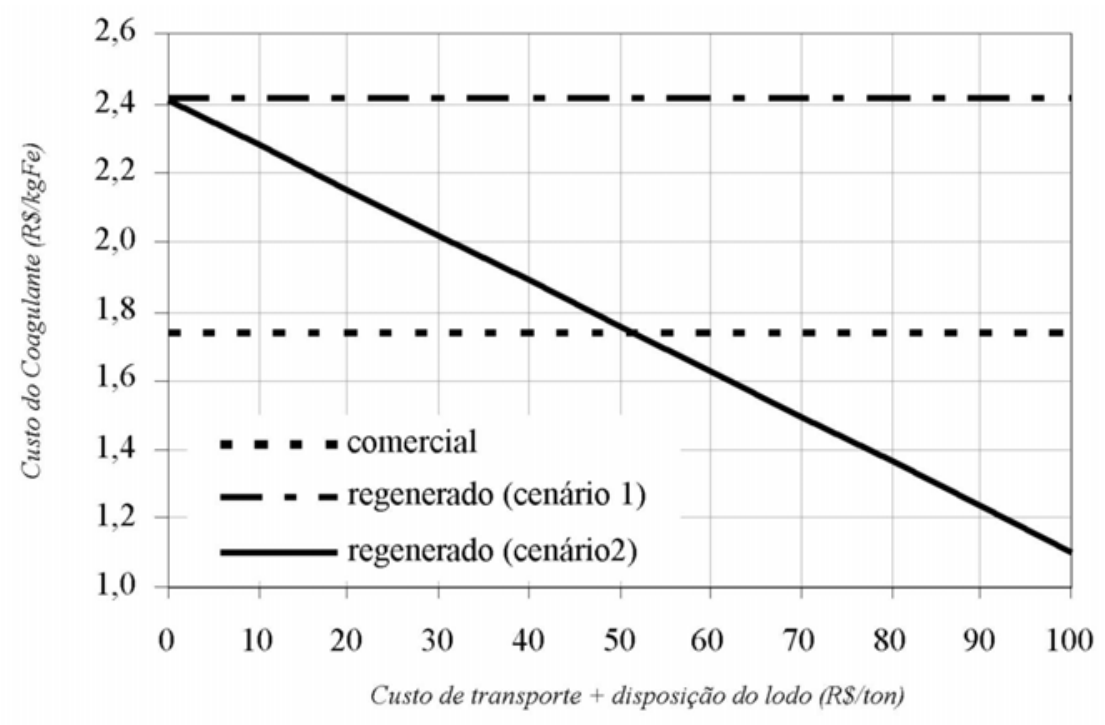

\section{Figura 8 - Variação do custo do coagulante com o custo de transporte e disposição do lodo (maio/04)}

Observando a Figura 8 percebe-se que o custo do coagulante regenerado pela simulação realizada no cenário 2 assume valor igual ao do coagulante comercial quando o custo de transporte e disposição do lodo é da ordem de $\mathrm{R} \$ 50,00 /$ ton, e, para valores mais altos que esse a operação do sistema de regeneração se torna economicamente viável.

Portanto, essa é uma variável extremamente significativa na avaliação econômica, e, em cada caso específico essa variável pode assumir valores muito distintos, interferindo na viabilidade econômica da tecnologia de regeneração de coagulantes.

É importante lembrar que os resultados apresentados são específicos para as condições observadas na ETA Rio Grande, sendo que a tecnologia pode ser viável ou não dependendo das características da ETA, tais como qualidade da água bruta, tipo de coagulante, dosagem de coagulante e localização da ETA, que interfere no custo de transporte e disposição do lodo.

Assim, a regeneração do coagulante pode ser viável economicamente ou não, dependendo das condições específicas de cada caso, pois em cada situação as variáveis técnicas relativas às condiçôes de operação e as às variáveis econômicas como custo do ácido sulfúrico e de transporte e disposição do lodo serão distintas.

Mas os resultados obtidos indicaram que a regeneração de coagulante pode ser viável, e isso justifica que seja considerada e estudada para cada caso específico, visando determinar onde pode ser apli- cada de forma viável economicamente. Além disso, mesmo que os benefícios econômicos não sejam muito significativos, deve se considerar que essa é uma alternativa ambientalmente adequada, uma vez que reduz a quantidade de resíduos e reaproveita um recurso.

\section{CONCLUSÕES}

Em relação aos aspectos técnicos da regeneração de coagulante e ao estudo de avaliação econômica, destacam-se as seguintes conclusōes:

- A realização de ensaios de bancada pode ser uma forma adequada para se determinar as condições ideais de acidificação, como $\mathrm{pH}$ e tempo de acidificação, para diferentes lodos.

- Os dois lodos apresentaram comportamento diferente em alguns aspectos, como concentração de contaminantes e redução da massa. Portanto, há necessidade de estudos em escala de bancada ou escala piloto para determinação dessas variáveis para cada lodo.

- Os coagulantes obtidos pela regeneração apresentaram concentraçōes de ferro da ordem de 100 vezes menores em relação aos valores usualmente presentes nos coagulantes comerciais.

- As concentrações de contaminantes obtidas nas análises dos coagulantes regenerados permitiram observar que os valores variavam significativamente com a ETA da onde foi retirado o lodo, provavelmente devido a diferenças na qualidade da água bruta e dos coagulantes utilizados, e, portanto, as concentrações de 
contaminantes devem ser avaliadas para cada caso específico.

- De modo geral, o coagulante regenerado a partir do lodo da ETA Rio Grande apresentou concentrações de contaminantes da mesma ordem de grandeza que os coagulantes comerciais, em relação à quantidade de ferro presente.

- O desempenho do coagulante regenerado nos ensaios com efluente de reator UASB foi equivalente ao obtido com coagulante comercial.

- Nas condições de regeneração ótimas determinadas, as reduçōes médias de sólidos em suspensão obtidas para o lodo da ETA Guaraú e ETA Rio Grande foram $28 \%$ e $53 \%$, respectivamente.

- Para as condiçōes consideradas, o custo do coagulante regenerado foi $28 \%$ maior que o do coagulante comercial quando não foi considerada a redução de custos decorrente da redução de lodo.

Considerando a redução dos custos propiciada pela redução de lodo, nas condiçôes adotadas, o custo do coagulante regenerado foi $6 \%$ inferior ao custo do coagulante comercial.

Considerando os aspectos apresentados anteriormente, a regeneração de coagulante é uma alternativa tecnicamente viável para os lodos estudados, produzindo um coagulante que pode ter aplicação em sistemas que não tenham como objetivo o tratamento de águas para abastecimento, como no tratamento de esgotos sanitários. Além disso é uma alternativa que pode ser economicamente viável, dependendo de características específicas de cada caso.

Assim, recomenda-se que ETA's já em funcionamento ou em fase de projeto que estejam em busca de alternativas para o lodo gerado na etapa de tratamento, considerem essa alternativa, e avaliem a viabilidade de aplicação para a sua situação específica.

\section{AGRADECIMENTOS}

A todos da SABESP - ETA Guaraú e ETA Rio Grande - por fornecerem a matéria prima da pesquisa e muitas informaçôes.

À Marycel Cotrim e à Profa ${ }^{a} r^{a}$ Maria Aparecida Faustino Pires, do IPEN - CQMA, pela realização de análises químicas que permitiram a obtenção, de maneira mais precisa, de dados fundamentais para a realização desse estudo.

Ao Wanderley Ferreira da Kemwater Brasil e ao Silvio Parisi da SABESP, por todas as informações e colaboração.
Aos funcionários do Laboratório de Saneamento e do Departamento de Engenharia Hidráulica e Sanitária, pelo suporte.

\section{REFERÊNCIAS}

APHA, AWWA, WEF. Standard Methods for the Examination of Water and Wastewater. EUA: 1995.

ASCE, AWWA, EPA. Management of Water Treatment Plant Residuals. EUA: 1996.

AWWA. Water Quality and Treatment. EUA: McGraw-Hill, $5^{a}$ edição, 1999.

AWWA. Coagulant Recovery: A Critical Assessment. EUA: AWWA Research Foundation, AWWA, 1991.

AWWA. Water Treatment Plant Waste Management. EUA: AWWA Research Foundation, 1987.

BISHOP, M.M. et al. Testing of Alum Recovery for Solids Reduction and Reuse. American Water Works Association Journal, EUA, Vol. 79, n06, Jun. 1987.

CORNWELL, D.A.; LEE, R.G. Waste Stream Recycling: Its Effect on Water Quality. American Water Works Association Journal. EUA, Vol. 86, $\mathrm{n}^{\circ} 11$, Nov. 1994.

FERREIRA FILHO, S.S.; ALÉM SOBRINHO, P. Consideraçôes sobre o tratamento de despejos líquidos gerados em estações de tratamento de água. Revista Engenharia Sanitária e Ambiental, v.3, n.4, 1998.

GONÇALVES, R.F.; PIOTTO, Z.C.; RESENDE, M.B. Influência dos Mecanismos de Coagulação da Água Bruta na Reciclagem de Coagulantes em Lodos de Estaçốes de Tratamento de Água. In: $19^{\circ} \mathrm{CON}-$ GRESSO DE ENGENHARIA SANITÁRIA E AMBIENTAL. ABES - ASSOCIAÇÃO BRASILEIRA DE ENGENHARIA SANITÁRIA E AMBIENTAL. Foz do Iguaçu, 1997.
MASIDES, J.; SOLEY, J.; MATA-ALVAREZ, J. A Feasibility Study of Alum Recovery in Wastewater Treatment Plants. Water Research. Grã Bretanha, Vol. 22, nº4, pg. 399-405, 1988.

MASSCHELEIN, W. J; DEVLEMINCK, R. GENOT, J. The Feasibility of Coagulant Recycling by Alkaline Reaction of Aluminum Hydroxide Sludges. Water Research. Grã Bretanha, Vol. 19, n ${ }^{\circ} 1$, pg. 1363-1368, 1985.

PETRUZELLI, D et al. Coagulants Removal and Recovery from Water Clarifier Sludge. Elsevier Science. Grã Bretanha, Vol. 34, nº 07, pg. 2177-2182, 2000.

PROSAB. Residuos Sólidos do Saneamento Processamento, Reciclagem e Disposição Final. Curitiba: Suprema Gráfica e Editora, $1^{\mathrm{a}}$ edição, 2001.

SABESP. RAP - Relatório Ambiental Preliminar: Aterro Exclusivo para Disposição de Lodo da ETA Taiaçupeba. São Paulo, 2004a.

SABESP. Sistemas de Captação e Tratamento Região Metropolitana de São Paulo. Disponível em <http://www.sabesp.com.br>. Acesso em $13 / 10 / 2004 b$

Endereço para correspondência:

Juliana Gardenalli de Freitas

Departamento de Engenharia

Hidráulica e Sanitária

Escola Politécnica da Universidade

de São Paulo

Av. Prof. Almeida Prado, Travessa,

$2-n^{\circ} 27$ I

05508-900 São Paulo - SP - Brasil

Tel.: (I I) 309 I-5220/309 I-5444

Fax: (I I) 309I-5423

E-mail:ssffilho@usp.br 